\title{
Recurrent erythema nodosum and pulmonary sarcoidosis
}

\author{
J. T. MACFARlane \\ M.R.C.P. \\ City Hospital, Hucknall Road, Nottingham NG5 IPB
}

\begin{abstract}
Summary
The case is described of a female patient who has had 3 separate episodes of erythema nodosum, hilar lymphadenopathy and pulmonary infiltration due to sarcoidosis over 20 years, with complete remissions in between. This has not been reported before and it raises questions about the aetiology of sarcoidosis.
\end{abstract}

\section{Introduction}

Erythema nodosum (EN) is usually associated with an acute form of sarcoidosis. Although EN sometimes reappears a few months after initial presentation (James, 1961), recurrences of EN and pulmonary sarcoidosis after remission are extremely unusual. A case is presented of 3 distinct episodes of EN and sarcoidosis of the lungs.

\section{Case report}

In March 1959, a 36-year-old woman was referred with a 2-month history of EN and a chest X-ray showing bilateral hilar lymphadenopathy (BHL), enlarged paratracheal lymph nodes and extensive bilateral nodular pulmonary infiltration. The Heaf test was weakly positive (grade I). A diagnosis of sarcoidosis was made. The EN resolved quickly and chest $\mathrm{X}$-rays showed progressive regression without treatment. By August 1960 the chest X-ray was normal and remained so until she was discharged from follow-up in 1962.

In March 1976, she had a recurrence of severe EN. She also developed shortness of breath. A chest X-ray showed BHL and extensive bilateral nodular infiltration. The ESR was $58 \mathrm{~mm} / \mathrm{hr}$ and a Heaf test was negative. Biopsy of the EN showed dermal and intradermal granulomas consistent with florid sarcoidosis. Lung function tests showed restrictive changes with lung volumes and transfer factor about $70 \%$ of predicted. The EN resolved and the chest $\mathrm{X}$-rays gradually cleared without treatment. By April 1978 the chest X-ray was normal. In October 1979, aged 56, she developed extensive EN again. She also had a dry cough and the chest X-ray again showed large BHL, enlarged paratracheal nodes and bilateral nodular pulmonary infiltration. Her ESR was $47 \mathrm{~mm} / \mathrm{hr}$ and her lung volumes had deteriorated.

Prednisolone $15 \mathrm{mg}$ daily was started. The EN disappeared over several weeks and her chest X-ray and dynamic lung volumes progressively improved as the corticosteroids were reduced slowly. Since
July 1980, she has been off corticosteroids and her chest X-ray has remained normal.

\section{Discussion}

This is the first report of a patient having 3 separate episodes of erythema nodosum, hilar lymphadenopathy and pulmonary infiltration due to sarcoidosis over 20 years, with complete remissions in batween. Recurrent EN has been reported once before in sarcoidosis (Scadding, 1967). The patient's chest X-ray during one of the attacks of EN showed slight BHL. Although relapse of sarcoidosis whilst reducing steroid therapy is well recognized, recurrence of pulmonary sarcoidosis after spontaneous cl zaring is extremely rare with only 3 other reported cases (Lim, 1951; Scadding, 1967; Symmons and Woods, 1980). EN occurs in up to one third of patients with sarcoidosis, the incidence varying from country to country, and is associated with a good prognosis. EN associated with sarcoidosis most commonly affects women of child-bearing age and its appearance after the age of 50 years, as it did twice in the present patient, is unusual (Scadding, 1967). The stimulus to the development of sarcoidosis is not known. Circulating free immune complexes appear in the serum during the early stages of EN and sarcoidosis (Verrier Jones et al., 1976), perhaps reflecting an unidentified antigen challenge or an intrinsic immune abnormality. However, it was not possible to identify any precipitating factor for the occurrence on 3 occasions of EN and pulmonary sarcoidosis in the present patient.

\section{Acknowledgment}

I thank Dr W. H. Roderick Smith for encouraging me to report this case under his care and for reviewing the chest $\mathrm{X}$-rays with me.

\section{References}

JAMES, D.G. (1961) Erythema nodosum. British Medical Journal, 1, 853.

LIM, K.H. (1961) Four episodes of sarcoidosis after pulmonary tuberculosis. Tubercle, 42, 350.

SCadding, J.G. (1967) Sarcoidosis. Eyre and Spottiswoode, London.

Symmons, D.P.M. \& Woods, K.L. (1980) Recurrent sarcoidosis. Thorax, 35, 879.

Verrier Jones, J., Cumming, R.H., Asplin, C.M., Laszlo, G. \& White, R.J. (1976) Circulating immune complexes in erythema nodosum and early sarcoidosis. British Medical Journal, 1, 153. 